\title{
Reasons for Qualitative Psychologists to Share Human Data
}

\author{
Veli-Matti Karhulahti ${ }^{1}$ \\ ${ }^{1}$ University of Jyväskylä, Faculty of Humanities and Social Sciences, Finland
}

\begin{abstract}
Author Note
Draft version 2.0, 28.09.2021. This draft has not been peer reviewed. Please find the final published version open access in the British Journal of Social Psychology: https://doi.org/10.1111/biso.12573.

I have no known conflict of interest to disclose.

Correspondence concerning this article should be addressed to Veli-Matti Karhulahti (vmmkar@utu.fi).
\end{abstract}

\begin{abstract}
Qualitative data sharing practices in psychology have not developed as rapidly as those in parallel quantitative domains. This is often explained by numerous epistemological, ethical, and pragmatic issues concerning qualitative data types. In this essay, I provide an alternative to the frequently expressed (often reasonable) concerns regarding the sharing of qualitative human data by highlighting three advantages of qualitative data sharing. I argue that sharing qualitative human data is not by default "less ethical," "riskier," and "impractical" compared to quantitative data sharing, but in some cases more ethical, less risky, and easier to manage for sharing because 1) informed consent can be discussed, negotiated, and validated, 2) the shared data can be curated by special means, and 3) the privacy risks are mainly local instead of global. I hope the provided alternative perspective further encourages qualitative psychologists to share their data when it is epistemologically, ethically, and pragmatically possible.
\end{abstract}




\section{Introduction}

In psychology, despite the ongoing replication crisis and increasing openness calls, keeping qualitative datasets closed is still the default option and rarely demanded, for instance, by funders, journals, or review boards. Compared to quantitative data sharing (e.g., Tedersoo et al. 2021), the opening of qualitative datasets has lagged due to numerous epistemological, ethical, and pragmatic issues (Broom et al. 2009; Kapiszewski \& Karcher 2020; Mauthner \& Parry 2009; Neale \& Bishop 2011). When it comes to human data-data regarding human individuals with which psychological sciences typically operate - various risks related to the qualitative nature of participation are commonly cited as barriers that warrant extreme attention and care, and ultimately, leading most researchers to err on the side of cautious non-sharing (for recent developments, see Alexander et al. 2020; Chauvette et al. 2019; Feldman \& Shaw 2019).

On the other hand, as Bishop (2009) observes, "most codes prescribe that participants should be exempt from unnecessary intrusion [and] if data already exist to address a research question, further collection of primary data could be seen as intrusive" (p. 259). This concerns elusive populations in particular, the study of which is often laborious for both researchers and participants (see Grinyer 2009; Long-Sutehall et al. 2010). Likewise, considering that the main reason for many people to participate in research as volunteers is the "wish to advance science" (Kuula 2011; see also Bourne and Robson 2015), not utilizing their contributions optimally can also be considered, in some instances, unethical or at least negligent (see Clark 2010). Participants should not be perceived solely as vulnerable subjects, but rather as actors with agency to be respected (see Schulz 2020). These examples speak for a perspective from which, when the data allows, they should be subject to similar sharing expectations that currently apply to quantitative datasets in many funding institutions, scientific journals, and review boards.

To shed further light on the above perspective, in this essay I provide an alternative to the frequently expressed (often reasonable) concerns regarding the sharing of qualitative human data. Starting with the premise that many forms of such data do involve distinct risks that need special attention and careful management, I shed light on three distinct viewpoints that demonstrate how the (collection of these) data also involves unique advantages in data sharing.

1) Informed consent can be discussed, negotiated, and validated.

2) Shared data can be curated.

3) Privacy risks are mainly local instead of global.

To be clear, my goal is not to produce new guidelines or recommendations. Rather, what the argued viewpoints collectively illustrate is that sharing qualitative human data is not by default "less ethical," "riskier," and "impractical" compared to quantitative data sharing, but in some cases, qualitative human datasets can be more ethical, less risky, and easier to manage for sharing. To clarify my terminology too, what follows applies explicitly to those qualitative human data that are produced collaboratively by the researcher and the participant in dynamic social contexts. Although I focus on interview and focus group data that are common in psychological sciences (with some references drawn from other qualitative approaches too), these data types occur in almost all social sciences and beyond, thus making the discussion relevant across fields. The space does not allow discussing other types of data (collection), which I look forward to being elaborated on in future studies. 
In an experiment by the Finnish Social Science Archive ten years ago (Kuula 2011), the scholars re-contacted 169 previous research participants who had shared their life stories and personal views. After asking for the participants' permissions to share the related qualitative data post hoc, 165 consented to share. Although one experiment (with four unique datasets) does now allow full generalization, it does imply that perhaps the biggest barriers for qualitative data sharing do not consist of epistemological, ethical, or pragmatic issues, but rather from the infrequent efforts of the researchers. This essay suggests three more reasons for qualitative researchers to consider exerting effort on sharing their data when it is epistemologically, ethically, and pragmatically possible.

\section{Sharing qualitative human data}

Qualitative human data have been shared for more than a hundred years; for instance, anthropologists have shared fieldnotes, films, and photographs since the birth of their field (see Broom et al. 2009; Mauthner \& Parry 2009; Wutich and Bernard 2016). However, the current standards of scholarly ethics differ from the times of early anthropology, and health and other types of sensitive data, which are commonly part of psychological research, entail several data management steps that can make their sharing practically impossible for many qualitative researchers. Although new infrastructures and knowledge regarding qualitative data sharing have been steadily emerging for a long time (e.g., Corti 2005), the challenges are still considered insurmountable to a degree that very few qualitative datasets in human research end up being shared (Tsai et al. 2016) let alone reused (Bishop \& Kuula-Luumi 2017). Numerous challenges have been identified in qualitative human data sharing (for a review, see Bishop 2009), but the present essay focuses on three, arguably the most central ones: consent, content, and risks.

\subsection{Consent as a challenge}

Although informed consent remains the "cornerstone" of human research ethics (Xu et al. 2020), plenty of criticism has emerged regarding the concept throughout decades. In an insightful discussion of the ethical dilemmas in qualitative family research, Larossa and colleagues (1981) list issues related to informed consent. Primarily, because qualitative research typically involves several unstructured elements, the participants cannot be informed completely of what the study involves. For instance, unexpected themes may emerge in semi-structured interviews, and the interview setting itself may involve events (like an interrupting phone call) that lead the interviewee to reveal unconsented information. Because the analysis of qualitative data is often exploratory, the final findings may also turn out surprising to a degree that potentially give some participants second thoughts about their involvement. While all these points apply to qualitative research in general, each concern remerges in sharing, as researchers assess whether the participant was informed properly of what is being shared.

Following the above, Richards and Schwartz (2002) discuss qualitative health research and highlight the need for consent to be managed as a "process" rather than a single event. They refer to the abovementioned unexpected elements of qualitative research practice and propose keeping in contact with participants so that they have the option to withdraw if new details regarding the study manifest, or if they for any reason consider the reuse of their stored data harmful. In qualitative studies, participants can sometimes be involved also in the planning, implementation, and dissemination of research, but consenting to such processes is not always 
straightforward. As Kaiser (2009) notes, many researchers lack a standardized process for obtaining additional consent, for which it is unlikely to be obtained. She stresses the need to inform that participants of the diverse foreseeable uses of the data as well as their possible reusers and readers, and as a solution, suggests an approach to consent where the participants are provided with multiple consent options. This naturally complicates the management of consent throughout the study and communicating such complexities to ethics committees remains a common challenge to qualitative researchers (e.g., Smette 2019).

In a recent interview study with eight scholars having varying experiences with shared qualitative data, Branney and colleagues (2019) illustrated the operation of dynamic consent in practice and identified consent as one of the key themes in qualitative data sharing. As an implication, they encourage data-sharing researchers to take into consideration the specific type of data and their options of sharing (e.g., different types come with different risks), the stakes and accountabilities of both participants and researchers (e.g., how does the relationship affect consenting), and the optimal means for negotiating consent with the participants (e.g., at which steps of the research process). These domains are revisited during secondary analysis, as researchers assess whether their motivations regarding the data cohere with the participants' consent. Taken together, the above nuances demonstrate how consent management in qualitative research entails care and ongoing consideration, both in sharing and reusing the datasets.

\subsection{Consent as an advantage}

As Gallagher and colleagues (2010) point out with a focus on child participants, those who are studied not always read let alone understand the consent form, and the same applies to caregivers or parents who are often nominated as gatekeepers by legal requirements. This, one may add, is an issue specifically prominent in (often quantitative) survey research, which are nowadays standardly participated via online platforms that enable immediate access without proper understanding of the consented participation. A study on online shoppers, for instance, found only one or two of every 1000 accessing the license agreement (Bakos et al. 2014). The societal and technological developments over the past decades (the internet) have changed the idea of what it means to "consent" in qualitative research, too: while people are increasingly sharing personal information publicly, being able predict what happens to it has become virtually impossible. Miller and Boulton (2007) observed this 15 years ago: "experiences of agency, power and risk all shape the qualitative research encounter in ways which cannot be anticipated by or encapsulated in information sheets or signed consent forms" (p. 2209).

Following the above, the scientific discussion regarding consent in the qualitative domain has almost exclusively focused on challenges and problems. Compared to other methodological orientations, however, there also several benefits in the consent procedures of qualitative human data collection, which should be given more attention especially in data sharing: when the participants and researchers operate in close social contact, consent can take a deep, dynamic, and a more transparent form. Instead of relying on a one-off instance where the participant is provided (large amounts of complex) information about their rights and the future usage of the data, the researcher can

a) discuss the forms of consent provided for the participant,

b) (re)negotiate the consent along with the development of the study, and

c) validate the nature of the consent at the end of the study, if applicable. 
In survey and similar research where the participants are in indirect contact with the research team, the situation rarely encourages direct human contact, especially if the default option of participation is anonymity. By contacting the research team, the participant exposes their identity, which may already exceed the critical limit if, for example, the requirements for inclusion relate to sensitive themes (e.g., having a specific illness or traumatic experience). In qualitative research, in turn, where the participant has already decided to enter a social situation with the researcher, complete anonymity has already been overcome and a degree of trust is to be established. This enables the researcher to ensure that the participant is properly informed and understands the information, while the participant can also ask further questions and affirm their will to participate. As such, consent in social qualitative settings can be more genuine; participants may withdraw after being better informed, and instead of needing to interpret written (often legal) text, they (should) have the opportunity make the researcher explain. Non-verbal communication can be relevant in assessing the participant's feelings, for instance, in cases when participants might be embarrassed or for other reasons cannot verbally communicate their will.

The openness of the qualitative situation further allows negotiating and renegotiating the discussed consent, assuming the participant is not anonymized after initial data collection. In the spirit of "consent as a process" (Richards and Schwartz, 2002), strategies of continuous consent are gathering increasing acceptance, focusing more on the respect of participant autonomy in the research relationship (Stutchbury \& Fox, 2009). In a recent example, Klykken (2021) illustrates these strategies by showing how "reflexive engagement with the principle of informed consent [is] done through the situated ethical work of explicitly and implicitly (re)negotiated consent and dissent" (p. 13). During the research, participants may signal explicitly and implicitly their willingness to keep sharing (certain) details, which the researcher can thus negotiate with the participant to exclude and include along with development of the study. Instead of invoking the printed words in a consent form, qualitative human researchers often have the option to update consent in the light of new information.

Finally, following Kaiser's (2009) idea of the "post-interview confidentiality form," qualitative researchers may also validate the participant's consent at the end of the study when all (potentially unexpected) information regarding the study have been collected and analyzed. This includes the details regarding the sharing of the data, which may (even with a careful data management plan) evolve during the research process. This is an opportunity to supplement the participant with the evolved details of the study and the role of their data in it. Of note, all the above negotiations, if planned to be done, are recommended to be agreed by the participant at the beginning of research, as continuous interaction can also be interpreted as making further demands on them (Grinyer 2009). For those participants who wish to be comprehensively informed about their participation and data sharing, the qualitative research settings can provide a more ethically robust platform of consent.

\subsection{Content as a challenge}

For a long time, qualitative data have been considered epistemologically different from quantitative data forms (e.g., Morgan \& Smircich 1980). For instance, some qualitative scholars believe that "researcher distance or neutrality is not only impossible, but completely defeats the epistemological purpose ... you are-according to this view-supposed to know what it feels

like rather than simply act as a detached witness" (Mason 2002, p. 92). Unlike in quantitative 
research where "distance from the data" is often considered an epistemological benefit, in some qualitative research distance can undermine the method. As these two positions are not always mutually exclusive-e.g., different epistemologies may be useful at different stages of research - both sides need to be considered in secondary analyses.

A frequent argument of those working with qualitative human data is that secondary use could be blocked (or at least complicated) by the re-user's lack of contextual knowledge, which can only be held by the original, data-collecting researcher (see e.g., Berg 2008; Moore 2007). For instance, body language that can be obvious for the interviewing researcher may be impossible to include in the transcript that is generated by an assistant based on an audio recording. For such qualitative content to be worthwhile in scientific reuse, careful data processing is required in collaboration with the collecting researcher and other involved parties.

Another aspect that separates qualitative human data from most quantitative data forms is the potentially unpredictable "intimacy" or "sensitivity" of content (e.g., Kuula 2011). Whereas the nature of quantitative data can be predefined somewhat clearly by closed-ended response options, open-ended responses give the participants the option to share delicate and highly personal information to a different degree. Disclosing private details regarding one's health, political opinions, and sexuality, among other such themes, yields data that are usually subject to special management requirements. Although the same regulatory conditions apply to quantitative data, (pre)determining the level of sensitivity in qualitative human data is generally more difficult due to its less structured nature (see e.g., Connor et al. 2018).

As a result of the above, many experts remain skeptical about qualitative data sharing. For instance, Broom and colleagues (2009) carried out an interview study regarding qualitative data archiving/sharing with 37 related experts, and one of their three key findings was specifically a group of concerns related to the privacy of information. In addition to the information associated with the participants' privacy, the experts also voiced issues regarding the privacy and rights of their own, namely, their role as co-producers of all data. Taken together, qualitative human data, due to their less controllable form, are generally considered involving more interpretive and regulatory challenges in reuse.

\subsection{Content as an advantage}

In human data sharing, it is common to remove participant details such as exact age and residency that, when combined, significantly contribute to the potential identification of the participants (e.g., Chalmers \& Muir 2003; Wiles et al. 2008). In research where the participants are not interacted directly with, as in online surveys, the removal of such details remains one of the few means to protect participants from being identified when sharing the datasets. In qualitative human research, in turn-where the researcher and participant (should) have established trust in direct social context - the data may be collaboratively curated to find the most optimal shape for sharing. As the saying goes, qualitative data are not collected but created. Although not all research designs allow collaborative engagement with the participants, such efforts have become a standard asset for many qualitative studies.

In focus groups and interviews, a general term covering most of the above co-curatorial work is "member checking" (e.g., Birt et al. 2016; Harvey 2015; Tong et al. 2007). By contacting the participants after initial data collection, the researchers can supplement, triangulate, and validate the data with the participants' follow-up input, which may also contribute to the data sharing process. For instance, transcribed documents can be returned to the participants for 
assessing the quality of the transcripts and making sure that details the participants do not wish to share will not be included as such in the archived documents. A case in Carlson's (2010) member checking illustrates:

When I received the checked transcripts back from Barry, I discovered he had made scores of editing notes, often correcting his original grammar. He had also added new items of information and deleted other things that I had thought were relevant, but apparently he did not (p. 1107)

In the above instance, Barry's edits were so major that the researcher had to re-consider how to interpret them in the light of the original data. At risk of stating the obvious, utmost care is needed in deciding how follow-up input will be taken into consideration (for a comprehensive scrutiny, see Goldblatt et al. 2011). Researchers and participants may choose to exclude some parts from the shared transcripts to protect a participant's identity from being revealed, yet apply the knowledge generated in those parts in their analysis. On the other hand, the participants may request some parts to be removed from all analysis, in which case researcher must assess the request against the negotiated consent, their personal understanding of the data, and the overall context in which knowledge is being co-produced (see Irwin 2013; Wiles et al. 2006). This is consistent with the previously discussed issues of epistemology in qualitative research. Sometimes knowledge is developed beyond the sharable data, and this must be accepted; nonetheless, being able to locate different epistemological levels-including sharable and unshareable sources of knowledge - is part of the qualitative researcher's interpretive work. The opportunity for the participants to further reflect on their own responses has also been found beneficial for the participants themselves (e.g., Harper \& Cole 2012).

In an oft-cited review of qualitative research validation strategies, Morse (2015) states unequivocally that the "researcher's background in theory and research methods must outrank the participant as a judge ... therefore, member checking as a strategy is not recommended" ( $p$. 1216). For Morse, however, the object of assessment is the validity of analysis and not the analyzed data as such. In data sharing, member checking remains a powerful tool that enables respecting the participant's evolving understanding of consent and working for solutions that, at the same time, allow the sharing of the valuable data for secondary use. Although straightforward removal of participant-selected details remains an option-the researcher must then assess case-by-case how they take this into consideration in analysis - it is also possible to negotiate alternative ways for expressing relevant information. In such instances, it is important to mark the shared transcripts accordingly, including meta-data, so that in reuse the edited or omitted parts can be distinguished and critically assessed. As Fielding (2000) noted already more than two decades ago: "it is very important that archived materials include as much information about the context of the original data collection as possible" (p. 23).

One means for reducing the risk for participant identification when sharing "as much as possible" is what I call data abstraction, i.e., replacing specific (potentially identifiable) details with more general terminology. For instance, a section in a transcript where a participant describes being 25 years old could be rephrased as "in their 20s" (with appropriate edit marking in the transcript). Because in qualitative human research how things are told typically represents the most important content, details such as exact ages, locations, names, times, and many others can often be abstracted with minimal loss. Even though similar strategies can be used in quantitative data sharing (e.g., turning exact respondent ages into age groups), the fundamental 
nature of statistical analyses (e.g., replicability-wise) and their lack of member checking options makes data abstraction generally more useful in qualitative research.

Again, many scholars might consider the above curatorial processes useless due to the fundamental epistemological inability for secondary users to understand the data in their full context. Referring to qualitative data sharing, Feldman and Shaw (2019) have recently expressed skepticism along these lines, being further afraid that "if viewed merely as discrete bits of information, any assessment of the claims made by the original author are vulnerable to being judged as inadequate" (p. 712). But it is exactly these calls for adequacy that have sparked scientific progress over the past centuries! Although qualitative methods are often singled out as operating with distinct epistemic principles, these principles cannot exclude critique, including that concerning (many kinds of) data when they serve as sources of evidence - all findings from research data are a construction that must be conveyable to others (Bishop 2006).

\subsection{Risks}

The risks related to research data can be divided in two connected but distinct domains: probability and damage caused to participants (see also Bahn \& Wetherill 2012). In qualitative human data sharing, the former is often considered to be greater than those in the quantitative domain. Parry and Mauthner (2004), for instance, support the above by describing how it is more difficult to conceal the identity of qualitative as opposed to quantitative research participants. As a result of this, the "measures taken to prepare qualitative data for archiving, including preservation of respondent anonymity and removal of key identifying characteristics, may compromise the integrity and quality of the dataset" (p. 148).

The above concerns dovetail the previously discussed concerns regarding context, which cannot be completely reproduced by an anonymized transcript or other documents that are always abstractions of the ephemeral multisensory data that one or more researchers coproduced. Even though current technology would enable storing and sharing these data in richer forms such as audio and video recordings, the risks they involve are typically deemed exceeding the benefits, not least because many regional guidelines consider such data automatically involving personal identifiers.

In concert with the discussed notion of qualitative content being "personal" or "sensitive" in a special way, it is possible that, in case of a security leak, the damages caused by unintended publicization of qualitative human data are also different. Such differences would likely be associated even more strongly with the specific type of content, however. In cases where the content poses an increased risk, researchers can give special attention to both the means of collecting and storing the data. Mealer and Jones (2014), for instance, used the telephone as their medium for interviewing individuals exposed to trauma, which allowed the participants to share their experiences without being visually exposed to the interviewer. Notably, similar risks of psychological stress also apply to the involved researchers who carry out heavy emotional work in processing sensitive human experiences as interviewers and in other roles (Dickson-Swift et al. 2007).

\subsection{Risks of different modes}

Despite the researchers' best efforts to secure "anonymity" to participants, it should be kept in mind that "anonymity" is not binary in practice but scales from strong to weak anonymity. 
Rocher and colleagues (2019) recently estimated that combining 15 attributes will be enough to reidentify an "anonymous" American respondent in a dataset with $99.98 \%$ accuracy, and much less attributes are needed for lower accuracy especially in groups such as the elderly, which can be relatively small. On the other hand, some scholars have argued that "it is more difficult to conceal the identity of qualitative as opposed to quantitative research participants [because] the level of detail in these combined datasets renders identification of respondents easy" (Parry \& Mauthner 2004, p. 144, 147, emphasis added).

Next to observations like the above that typically focus on the strengths and weaknesses of anonymity, the different modes of anonymity are less often discussed. In one useful study, Tolich (2004) separates "external confidentiality"- the default idea that researchers do not reveal participants' identities to externals without consent-from "internal confidentiality," i.e. the "ability for research subjects involved in the study to identify each other" (p. 101). The latter, while not limited to qualitative studies, represents a critical instance that scholars with qualitative human data struggle more often than their quantitative peers. To further illustrate these methodologically specific differences, I argue there is also a need to distinguish between local and global risks related to human data, the former of which is more (but not solely) associated with qualitative data and the latter with quantitative data.

By local risks I refer to that "level of detail," which many scholars and stakeholders consider problematic especially when it comes to sharing qualitative human data. Narratives about one's personal experiences, events that have taken place in a community, and curiosities that are known only by a few are examples where pieces of information can immediately reveal the participant's identity to a reader who belongs to a related small local group. Notably, these readers are not limited to those involved in the research environment (as in Tolich 2004). By numbers, the locals with the capacity to easily identify a participant of a qualitative study are very few and, in the long run, their number will unlikely increase due to the ephemeral and historical nature of the events that constitute the primary risk for revealing participants' identities. For example, if a participant shares details regarding an event that took place between three people, these three will remain as the group with a high probability to identity the participant, and the probability for this group to expand (e.g., by details being retold) is relatively small. Unless there are reasons to believe that the local individuals have special motivations to seek out the data-e.g., if the data concern victims of abuse or political targets-local risks can be significantly smaller than global ones.

By global risks, in turn, I refer to the (often demographic) attributes that participants of especially quantitative survey studies disclose as part of their participation. When such attributes are explicitly collected and listed for each participant in a shared dataset, the related risks are less about a selected group of (local) people being able to connect these data to the participant, and more about the available statistical methods being able to reconstruct a profile that anyone with the right tools can connect to the participant with a high probability. Because these methodological developments are rapid and unpredictable (Nature 2019), global risks are less temporally controllable; namely, a dataset with specific human attributes that has had strong anonymity in the past may well have weak anonymity today.

Of course, local and global risks are not mutually exclusive and all human datasets involve both to some degree. Nevertheless, the risks associated with qualitative human datasets, which are coproduced in social researcher-participant interaction, are distinctly more local than global. Even though these risks may be more immediate in the sense that the right local persons may have a high probability of being able to identify the participant, the number of these persons 
(and thus the probability of critical data access) is significantly smaller; moreover, the risks related to the development of new methodological means for combining participant attributes into identifiable profiles, based on recent developments, are of smaller scale. Accordingly, when discussing the risks related to sharing qualitative human data, it is important to recognize that the concept of risk is not merely a matter of "high" and "low" but also of mode, and in some instances, the local risk types common to qualitative data forms may be lesser than the global risks related to many quantitative datasets.

\section{Conclusions}

The increasing calls, demands, and encouragements for data sharing have significantly improved the quality of research across psychological and other fields, resulting in higher levels of reliability, replicability, and transparency in general. While not all these improvements are relevant to qualitative researchers whose value concepts are partially different (e.g., Kapiszewski \& Karcher 2020; Stenbacka 2001), this should not discourage qualitative scholars from pursuing transparency in data sharing. Some forms of replicability do apply to qualitative research as well (see Goodman et al. 2016; Peels \& Bouter 2018), and as Bishop (2005) noted a long time ago,

the advancement of qualitative methods suffers when researchers ... believe their research is deficient and thus unworthy of being shared. In fact, transparency makes it possible to reveal the deep expertise and considered judgement researchers put into difficult choices ... New and experienced researchers alike can benefit from this kind of scrutiny of archival materials (p. 334-5)

So far, several pushbacks with epistemological, ethical, and pragmatic concerns have kept qualitative human datasets largely unshared. Many of these concerns are valid and scholars need to keep assessing the tradeoffs of sharing (all kinds of) data, usually on a case-by-case basis throughout an ethics of practice (Guillemin \& Gillam 2004). With respect to this sensitivity needed to assess the shareability of each dataset, my goal has not been to produce recommendations, but rather to highlight that sharing qualitative human data is not by default "less ethical," "riskier," and "impractical" compared to quantitative data sharing. Rather, in some cases, qualitative human datasets can be more ethical, less risky, and easier to manage for sharing. In this essay, the claim was supported by three qualitative data sharing advantages.

First, in qualitative human research, informed consent can be discussed, negotiated, and validated. Unlike in many quantitative forms of human data collection, the qualitative researcher can be in direct social contact with the participants, which allows consent to be discussed beyond "click to agree" forms, and along the way, further negotiated and validated. For those researchers who make use of these possibilities, this yields an ethical benefit that provides the data sharer more adjustable and reliable forms of participant consent. Sometimes, the negotiations may also lead to dissent, which serves as a valuable means to identify and avoid participant-specific risks - an option that is rarely available in quantitative data collection.

Second, the researcher-participant relationship also opens the possibility for member checking, which is an asset in data sharing. Parts that may not be comfortable for sharing can be marked by participants, which facilitates anonymization and adds to the ethics of participant autonomy and respect. Researchers can also use data abstraction in curation to reformulate details. Although modifying original data entails a risk of misinterpretation and losing something 
that future analysts deem significant, the increasing standards of presenting qualitative human data will protect re-users from epistemological pitfalls.

Third, the risks related to participant privacy in qualitative research are essentially local, i.e., the possible reidentification of participants would occur mainly by a small group of individuals who are familiar with the shared details. Even though these types of risks may be difficult to eliminate entirely, they rarely accumulate over time and their overall probability is relatively low due to the small numbers of possible re-identifiers. Compared to the global risks that many quantitative studies struggle with-demographic attributes allowing the reconstruction of the participant's identity by anyone with proper tools - it is not true that qualitative human data sharing is automatically riskier, but the risks and their management are simply different; not by default higher or lower, but case-specific.

As a limitation, the nature of this essay did not allow discussing multiple distinct data types and the methods of their collection. This may have rightly provoked some readers to ponder generalizability. That said, it is unlikely that qualitative research or data management can ever find one-size-fits-all solutions. Future research would do well to inform us about the nuances of sharing specific qualitative data types.

Finally, as the sharing of qualitative data does often differ significantly from other types of data sharing, future research should pursue more advanced methodological solutions and protocols, which can further help scholars to share their valuable qualitative data for reuse. Recently, many national archives have made major progress in supporting scholars with related challenges by offering their experience and infrastructures (Bishop \& Kuula-Luumi 2017); however, national archives tend to be limited to local use (e.g., for linguistic reasons). Efforts should be directed for building more global archival networks that enable scholars from all backgrounds and cultural groups equal opportunities for data sharing.

\section{References}

Alexander, S. M., Jones, K., Bennett, N. J., Budden, A., Cox, M., Crosas, M., ... \& Weber, N. (2020). Qualitative data sharing and synthesis for sustainability science. Nature Sustainability, 3 (2), 81-88.

Bahn, S., \& Weatherill, P. (2013). Qualitative social research: a risky business when it comes to collecting 'sensitive' data. Qualitative research, 13 (1), 19-35.

Bakos, Y., Marotta-Wurgler, F., \& Trossen, D. R. (2014). Does anyone read the fine print? Consumer attention to standard-form contracts. The Journal of Legal Studies, 43 (1), 1-35.

Berg, Van den H. (2008). Reanalyzing qualitative interviews from different angles: The risk of decontextualization and other problems of sharing qualitative data. Historical Social Research/Historische Sozialforschung, 179-192.

Birt, L., Scott, S., Cavers, D., Campbell, C., \& Walter, F. (2016). Member checking: a tool to enhance trustworthiness or merely a nod to validation? Qualitative Health Research, 26 (13), 1802-1811.

Bishop, L. \& Kuula-Luumi, A. (2017). Revisiting qualitative data reuse: A decade on. Sage Open, 7 (1), 2158244016685136.

Bishop, L. (2005). Protecting respondents and enabling data sharing: Reply to Parry and Mauthner. Sociology, 39 (2), 333-336.

Bishop, L. (2006). A proposal for archiving context for secondary analysis. Methodological Innovations Online, 1 (2), 10-20. 
Bishop, L. (2009). Ethical sharing and reuse of qualitative data. Australian Journal of Social Issues, 44 (3), 255-272.

Bourne, A. H., \& Robson, M. A. (2015). Participants' reflections on being interviewed about risk and sexual behaviour: implications for collection of qualitative data on sensitive topics. International journal of social research methodology, 18 (1), 105-116.

Branney, P., Reid, K., Frost, N., Coan, S., Mathieson, A., \& Woolhouse, M. (2019). A contextconsent meta-framework for designing open (qualitative) data studies. Qualitative Research in Psychology.

Broom, A., Cheshire, L., \& Emmison, M. (2009). Qualitative researchers' understandings of their practice and the implications for data archiving and sharing. Sociology, 43 (6), 1163-1180.

Campbell, B., Thomson, H., Slater, J., Coward, C., Wyatt, K., \& Sweeney, K. (2007). Extracting information from hospital records: what patients think about consent. BMJ Quality \& Safety, 16 (6), 404-408.

Candela, A. G. (2019). Exploring the function of member checking. The Qualitative Report, 24 (3), 619-628.

Carlson, J. A. (2010). Avoiding traps in member checking. Qualitative Report, 15 (5), 11021113.

Chalmers, J., \& Muir, R. (2003). Patient privacy and confidentiality: The debate goes on; the issues are complex, but a consensus is emerging. BMJ 326, 725-6.

Chauvette, A., Schick-Makaroff, K., \& Molzahn, A. E. (2019). Open data in qualitative research. International Journal of Qualitative Methods, 18, 1609406918823863.

Clark, T. (2010). On 'being researched': Why do people engage with qualitative research? Qualitative Research, 10 (4), 399-419.

Connor, J., Copland, S., \& Owen, J. (2018). The infantilized researcher and research subject: Ethics, consent and risk. Qualitative Research, 18 (4), 400-415.

Corti, L. (2006). Qualitative archiving and data sharing: Extending the reach and impact of qualitative data. IASSIST Quarterly, 29 (3), 8-13.

Dickson-Swift, V., James, E. L., Kippen, S., \& Liamputtong, P. (2007). Doing sensitive research: what challenges do qualitative researchers face?. Qualitative research, 7 (3), 327-353.

Feldman, S., \& Shaw, L. (2019). The epistemological and ethical challenges of archiving and sharing qualitative data. American Behavioral Scientist, 63 (6), 699-721.

Fielding, N. (2000) 'The shared fate of two innovations in qualitative methodology: The relationship of qualitative software and secondary analysis of archived qualitative data', Forum: Qualitative Social Research, 1(3) Art. 22.

Gallagher, M., Haywood, S. L., Jones, M. W., \& Milne, S. (2010). Negotiating informed consent with children in school-based research: a critical review. Children \& Society, 24 (6), 471-482.

Goldblatt, H., Karnieli-Miller, O., \& Neumann, M. (2011). Sharing qualitative research findings with participants: Study experiences of methodological and ethical dilemmas. Patient education and counseling, 82 (3), 389-395.

Goodman, S. N., Fanelli, D., \& Ioannidis, J. P. (2016). What does research reproducibility mean? Science translational medicine, 8 (341), 341ps12-341ps12.

Grinyer, A. (2009). The ethics of the secondary analysis and further use of qualitative data. Social Research Update, 56 (4), 1-4.

Guillemin, M., \& Gillam, L. (2004). Ethics, reflexivity, and "ethically important moments" in research. Qualitative inquiry, 10 (2), 261-280. 
Harper, M., \& Cole, P. (2012). Member checking: Can benefits be gained similar to group therapy. The qualitative report, 17 (2), 510-517.

Harvey, L. (2015). Beyond member-checking: A dialogic approach to the research interview. International Journal of Research \& Method in Education, 38 (1), 23-38.

Irwin, S. (2013). Qualitative secondary data analysis: Ethics, epistemology and context. Progress in Development Studies, 13 (4), 295-306.

Kaiser, K. (2009). Protecting respondent confidentiality in qualitative research. Qualitative health research, 19 (11), 1632-1641.

Kapiszewski, D., \& Karcher, S. (2021). Transparency in practice in qualitative research. PS: Political Science \& Politics, 54 (2), 285-291.

Klykken, F. H. (2021). Implementing continuous consent in qualitative research. Qualitative Research, 14687941211014366.

Kuula, A. (2011). Methodological and ethical dilemmas of archiving qualitative data. IASSIST Quarterly, 34 (3-4).

LaRossa, R., Bennett, L. A., \& Gelles, R. J. (1981). Ethical dilemmas in qualitative family research. Journal of Marriage and the Family, 43 (2), 303-313.

Long-Sutehall, T., Sque, M., \& Addington-Hall, J. (2011). Secondary analysis of qualitative data: a valuable method for exploring sensitive issues with an elusive population? Journal of Research in Nursing, 16 (4), 335-344.

Mason, J. (2002). Qualitative Researching. Sage.

Mauthner, N. S., \& Parry, O. (2009). Qualitative data preservation and sharing in the social sciences: On whose philosophical terms? Australian Journal of Social Issues, 44 (3), 291-307.

Mealer, M., \& Jones, J. (2014). Methodological and ethical issues related to qualitative telephone interviews on sensitive topics. Nurse Researcher, 21 (4).

Miller, T., \& Boulton, M. (2007). Changing constructions of informed consent: Qualitative research and complex social worlds. Social Science \& Medicine, 65 (11), 2199-2211.

Moore, N. (2007). (Re) using qualitative data? Sociological Research Online, 12 (3), 1-13.

Morgan, G., \& Smircich, L. (1980). The case for qualitative research. Academy of Management Review, 5 (4), 491-500.

Morse, J. M. (2015). Critical analysis of strategies for determining rigor in qualitative inquiry. Qualitative Health Research, 25 (9), 1212-1222.

Nature (2019) Digital-data studies need consent. Editorial. Nature, vol. 572. Iss. 7767. DOI:10.1038/d41586-019-02322-z

Neale, B., \& Bishop, L. (2011). Qualitative and Qualitative Longitudinal Resources in Europe. IASSIST Quarterly, 34 (3-4).

Parry, O., \& Mauthner, N. S. (2004). Whose data are they anyway? Practical, legal and ethical issues in archiving qualitative research data. Sociology, 38 (1), 139-152.

Peels, R., \& Bouter, L. (2018). The possibility and desirability of replication in the humanities. Palgrave Communications, 4 (1), 1-4.

Richards, H. M., \& Schwartz, L. J. (2002). Ethics of qualitative research: are there special issues for health services research? Family Practice, 19 (2), 135-139.

Rocher, L., Hendrickx, J. M., \& De Montjoye, Y. A. (2019). Estimating the success of reidentifications in incomplete datasets using generative models. Nature Communications, 10 (1), $1-9$.

Schulz, P. (2020). Recognizing research participants' fluid positionalities in (post-) conflict zones. Qualitative Research, 1468794120904882. 
Smette, I. (2019). Ethics and access when consent must come first: consequences of formalised research ethics for ethnographic research in schools. In Implementing Ethics in Educational Ethnography (pp. 51-63). Routledge.

Stenbacka, C. (2001). Qualitative research requires quality concepts of its own. Management Decision. 39 (7), 551-556.

Tedersoo, L., Küngas, R., Oras, E., Köster, K., Eenmaa, H., Leijen, Ä., ... \& Sepp, T. (2021). Data sharing practices and data availability upon request differ across scientific disciplines. Scientific Data, 8 (1), 1-11.

Tolich, M. (2004). Internal confidentiality: When confidentiality assurances fail relational informants. Qualitative Sociology, 27 (1), 101-106.

Tong, A., Sainsbury, P., \& Craig, J. (2007). Consolidated criteria for reporting qualitative research (COREQ): a 32-item checklist for interviews and focus groups. International Journal for Quality in Health Care, 19 (6), 349-357.

Tsai, A. C., Kohrt, B. A., Matthews, L. T., Betancourt, T. S., Lee, J. K., Papachristos, A. V., ... \& Dworkin, S. L. (2016). Promises and pitfalls of data sharing in qualitative research. Social Science \& Medicine, 169, 191-198.

Verity, C., Manning, D., \& Nicoll, A. (2002). Consent, confidentiality, and the threat to public health surveillance commentary: don't waive consent lightly, involve the public. $B M J, 324$ (7347), 1210-1213.

Wiles, R., Charles, V., Crow, G., \& Heath, S. (2006). Researching researchers: lessons for research ethics. Qualitative Research, 6 (3), 283-299.

Wiles, R., Crow, G., Heath, S., \& Charles, V. (2008). The management of confidentiality and anonymity in social research. International Journal of Social Research Methodology, 11 (5), 417-428.

Wutich, A., \& Bernard, H. R. (2016). Sharing qualitative data \& analysis. With whom and how widely? A response to 'Promises and pitfalls of data sharing in qualitative research'. Social Science \& Medicine, 169, 199-200.

Xu, A., Baysari, M. T., Stocker, S. L., Leow, L. J., Day, R. O., \& Carland, J. E. (2020). Researchers' views on, and experiences with, the requirement to obtain informed consent in research involving human participants: a qualitative study. BMC Medical Ethics, 21 (1), 1-11. 